Swarthmore College

Works

Physics \& Astronomy Faculty Works

Physics \& Astronomy

2009

\title{
3D Reconnection And Flow Dynamics In The SSX Experiment
}

Michael R. Brown

Swarthmore College, doc@swarthmore.edu

C. D. Cothran

David H. Cohen

Swarthmore College, dcohen1@swarthmore.edu

Jason Alexander Horwitz , '07

Vernon Hampden Chaplin , '07

Follow this and additional works at: https://works.swarthmore.edu/fac-physics

Part of the Astrophysics and Astronomy Commons, and the Physics Commons

Let us know how access to these works benefits you

\section{Recommended Citation}

Michael R. Brown; C. D. Cothran; David H. Cohen; Jason Alexander Horwitz , '07; and Vernon Hampden Chaplin , '07. (2009). "3D Reconnection And Flow Dynamics In The SSX Experiment". Current Trends In International Fusion Research: Proceedings Of The 7th Symposium. Volume 1154, 167-176. DOI: 10.1063/ 1.3204572

https://works.swarthmore.edu/fac-physics/70

This work is brought to you for free by Swarthmore College Libraries' Works. It has been accepted for inclusion in Physics \& Astronomy Faculty Works by an authorized administrator of Works. For more information, please contact myworks@swarthmore.edu. 


\title{
3D RECONNECTION AND FLOW DYNAMICS IN THE SSX EXPERIMENT
}

\author{
M.R. Brown, C.D. Cothran, D.H. Cohen, J. Horwitz, and V. Chaplin \\ Department of Physics and Astronomy \\ Center for Magnetic Self Organization \\ Swarthmore College \\ Swarthmore, PA 19081-1397 U.S.A.
}

\begin{abstract}
Several new experimental results are reported from plasma merging studies at the Swarthmore Spheromak Experiment (SSX) with relevance to collisionless three-dimensional magnetic reconnection in laboratory and space plasmas. First, recent high-resolution velocity measurements of impurity ions using ion Doppler spectroscopy (IDS) show bi-directional outflow jets at $40 \mathrm{~km} / \mathrm{s}$ (nearly the Alfven speed). The SSX IDS instrument measures with 1 $\mu$ s or better time resolution the width and Doppler shift of the $\mathrm{C}_{\mathrm{III}}$ impurity ( $\mathrm{H}$ plasma) 229.7 $\mathrm{nm}$ line to determine the temperature and line-averaged flow velocity during spheromak merging events. High flow speeds are corroborated using an in situ Mach probe. Second, ion heating to nearly $10^{6} \mathrm{~K}$ is observed after reconnection events in a low-density kinetic regime. Transient electron heating is inferred from bursts on a 4-channel soft x-ray array as well as vacuum ultraviolet spectroscopy. Third, the out-of-plane magnetic field and the in-plane Lorentz force in a reconnection volume both show a quadrupolar structure at the ion inertial scale $\left(\mathbf{c} / \omega_{\mathrm{pi}}\right)$. Time resolved vector magnetic field measurements on a $3 \mathrm{D}$ lattice $\mathbf{B}(\mathbf{r}, \mathbf{t})$ ) enables this measurement. Earlier work at SSX has shown that formation of three-dimensional structure at the ion inertial scale is temporally and spatially correlated with the observation of superthermal, super-Alfvenic ions accelerated along the X-line normal to the local 2D plane of reconnection. Each of these measurements will be related to and compared with similar observations in a solar or space context. Keywords: spheromak, flow, heating
\end{abstract}

\section{INTRODUCTION}

Magnetic reconnection is the engine driving the dynamics in spheromak formation, relaxation, and merging[1,2]. During reconnection, magnetic energy is rapidly converted to electron and ion heat, as well as plasma flow. Spheromak dynamics have been studied at the Swarthmore Spheromak Experiment (SSX) in a number of geometries[3-6]. We have recently been studying spheromak merging in a prolate $0.4 \mathrm{~m}$ diameter, $0.6 \mathrm{~m}$ length, $3 \mathrm{~mm}$ wall copper flux conserver at SSX. Merging of a pair of counter-helicity spheromaks generates magnetic reconnection dynamics at the midplane. Injection of a single spheromak 
into a vacuum dipole magnetic field generates a quiescent, axisymmetric toroidal structure that is stable for the lifetime of the object, despite the elongated flux conserver

Local and global magnetic structure of SSX spheromaks has been studied with up to 600 individual internal magnetic probes operated simultaneously at $1.25 \mathrm{MHz}$ using a multiplexer system.[7] Line averaged electron density is monitored with a quadrature HeNe laser interferometer.[8] We can scan density in a range $n_{e}=1-10 \times 10^{14} \mathrm{~cm}^{-3}$. Line averaged electron temperature is inferred from a model fit to data from a soft x-ray array. SSX plasmas have temperatures $T_{e} \leq T_{i} \approx 80 \mathrm{eV}$, and typical magnetic fields of $0.1 \mathrm{~T}$. The plasma is fully ionized and fully magnetized $\rho_{\mathrm{i}}<\mathrm{R}$, where $\mathrm{R}=0.2-0.25 \mathrm{~m}$ is the outer flux conserving boundary of the plasma (defined by a cylindrical copper wall). The Lundquist number $\mathrm{S}$, the ratio of the resistive magnetic diffusion time $\tau_{\mathrm{R}}$ to the Alfven transit time $\tau_{\mathrm{A}}$, is large for SSX, $\mathrm{S} \approx 1000$. Accordingly, the global structure of SSX spheromaks is fully in the magnetohydrodynamic $(\mathrm{MHD})$ regime $\left(\mathrm{S}>>1, \rho_{\mathrm{i}}<<\mathrm{R}\right)$.

In addition, line averaged ion flow and temperature $\mathrm{T}_{\mathrm{i}}$ at the midplane is monitored with a $1.33 \mathrm{~m}$ ion Doppler spectrometer (IDS) for this configuration. We achieve high spectral resolution $(0.0075 \mathrm{~nm}$ per pixel) with an Echelle grating operating at 25 th order[9]. We achieve high temporal resolution by using a 32 channel photomultiplier tube array. The SSX IDS instrument measures with $1 \mu \mathrm{s}$ or better time resolution the width and Doppler shift of the $\mathrm{C}_{\text {III }}$ impurity (H plasma) $229.7 \mathrm{~nm}$ line to determine the temperature and line-averaged flow velocity during spheromak trapping. The instrument design temperature is approximately $3 \mathrm{eV}$ for C emission lines. Velocity resolution is about $5 \mathrm{~km} / \mathrm{s}$, corresponding to approximately 0.05 $\mathrm{v}_{\mathrm{A}}$. The Czerny-Turner spectrometer has $1.33 \mathrm{~m}$ focal length, $\mathrm{f} / 9.4$, and uses a 316 groove $/ \mathrm{mm}$ Echelle grating blazed for a $63.5^{\circ}$ angle. The $\mathrm{C}_{\mathrm{III}}$ line is observed at 25 th order where the spectrometer achieves a dispersion of $0.03 \mathrm{~nm} / \mathrm{mm}$. Line averaged IDS measurements are corroborated locally with a Mach probe.

In section II, measurements of the reconnection-driven outflow are discussed (both spectroscopic and probe measurements). In section III, measurements of reconnection-driven heating (electrons and ions) is presented. In section IV, measurements of the quadrupolar outof-plane magnetic field are presented. A summary is presented in section $\mathrm{V}$.

\section{RECONNECTION OUTFLOW}

The SSX apparatus uses opposing, coaxial magnetized plasma guns at either end of a cylindrical copper flux conserver to produce spheromaks of either handedness (see figure 1). Several different flux conservers have been used at SSX over the years with diameters varying between 0.17 and $0.5 \mathrm{~m}$. The present flux conserver diameter is $0.40 \mathrm{~m}$ with a length of 0.61 $\mathrm{m}$ (aspect ratio or elongation of 1.5 and $\mathrm{L} / \mathrm{R}=3$ ). Spheromak merging drives magnetic reconnection at the midplane. We expect unmagnetized plasma to be expelled from both sides of the reconnection region at the Alfven speed. We have made line-averaged measurements of this process with ion Doppler spectroscopy and local measurements with Mach probes.

Previously, we have measured the three dimensional structure of magnetic reconnection, [10] the production of beams of energetic ions accelerated by reconnection electric fields, [8] and various terms in the generalized Ohm's law within the reconnecting volume.[11] The scale of the reconnection current sheet in SSX is about $\mathrm{c} / \omega_{\mathrm{pi}}=2 \mathrm{~cm}[12]$ and Hall effects are large[11] leading us to believe two fluid effects are important. Local three dimensional structures have been studied with a high resolution magnetic probe array capable of measuring vector $\mathbf{B}$ on a $5 \times 5 \times 8$ grid, 200 simultaneous measurements of vector $\mathbf{B}$, at a spatial resolution of about the ion scale $\left(15 \mathrm{~mm}\right.$ radially and $18 \mathrm{~mm}$ axially which is $<\mathrm{c} / \omega_{\mathrm{pi}}$ in SSX).[7] Large scale magnetic structures have been studied at coarser resolution $(25 \mathrm{~mm}$ resolution radially) with up to 20 linear arrays threading the machine.[4] 


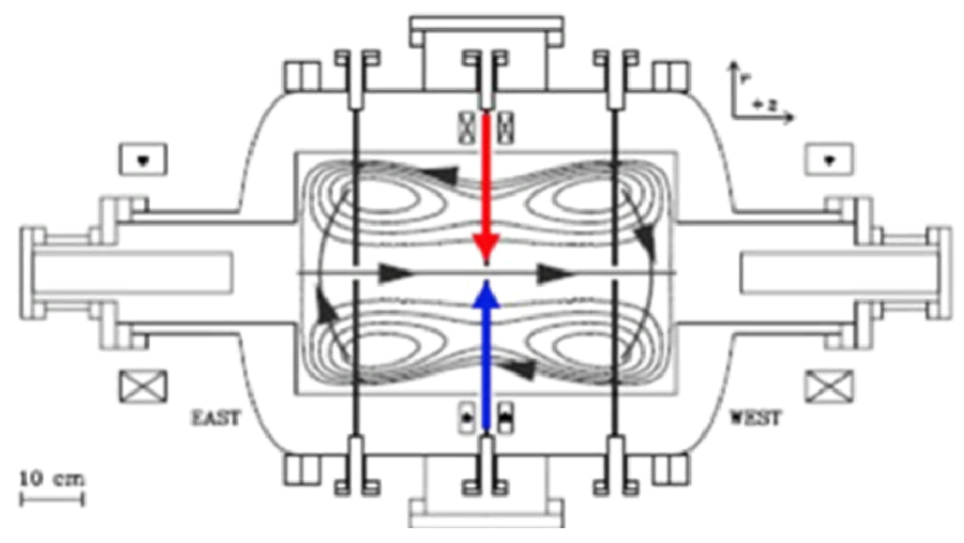

Figure 1. Swarthmore Spheromak Experiment. Lines of sight for IDS/SXR/VUV (midplane) and probe locations are depicted.

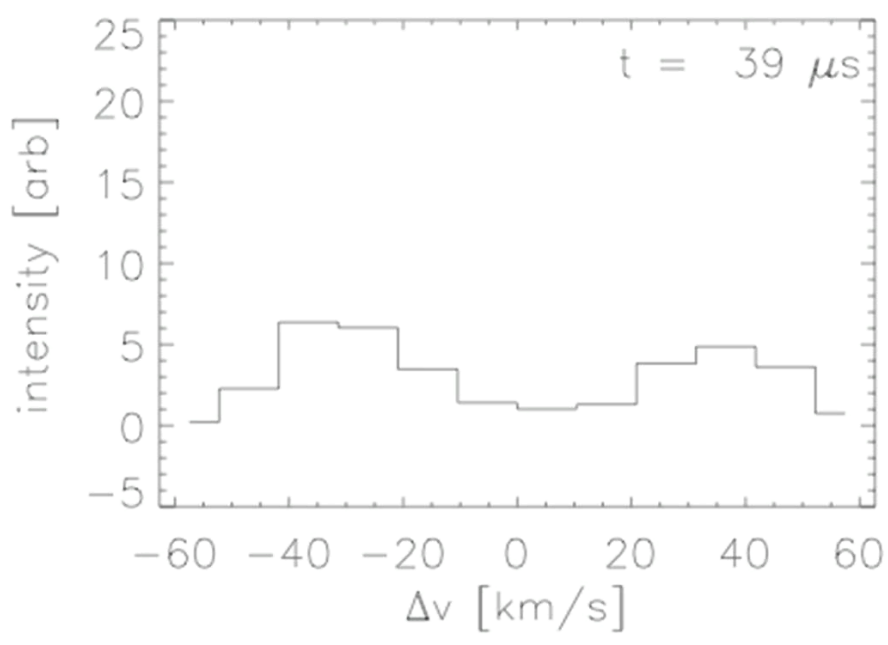

Figure 2. Outflow jets measured with ion Doppler spectroscopy. Dynamics of $\mathrm{C}_{\mathrm{III}}$ emission along a midplane chord midplane are depicted. Jets persist for several $\mu \mathrm{s}$ at $\pm \mathrm{V}_{\mathrm{A}}$.

A characteristic signature of reconnection dynamics is a relatively slow inflow into the reconnection volume and rapid, nearly Alfvenic outflow. The inflow speed is regulated in response to the outflow. The outflow should manifest itself as two oppositely directed jets orthogonal to the inflow direction. 


\section{A. Ion Doppler spectroscopy}

In figure 2, we show a lineshape from $\mathrm{C}_{\text {III }}$ emission measured during a single reconnection event. The chord for this sequence is across the full $0.4 \mathrm{~m}$ diameter at the midplane as shown in Figure 1. Motion both towards and away from the observer is evident. Simultaneous bi-directional outflow with velocities $\pm 40 \mathrm{~km} / \mathrm{s}$ is clearly evident at about 40 $\mu \mathrm{s}$.[13] An outflow jet of $40 \mathrm{~km} / \mathrm{s}$ corresponds to about $0.4 \mathrm{~V}_{\mathrm{A}}$ in SSX. Note that since the process is dynamic and fully $3 \mathrm{D}$, we observe bi-directional jets in only 1 in 5 shots.

\section{B. Mach probe}

A Mach probe is essentially a directional electrostatic ion collector measuring upstream and downstream ion current simultaneously. SSX Mach probes feature 3 pairs of opposing tungsten collectors housed in boron nitride sheath. The Mach number measured by the probe is equal to $\mathrm{M}=\kappa \ln \left(\mathrm{I}_{\text {up }} / \mathrm{I}_{\text {down }}\right)$ (where $\kappa$ is a proportionality constant). While ab initio calculation of the proportionality constant $\kappa$ is both complicated and controversial[14], SSX Mach probes are absolutely calibrated using a time-of-flight technique. Using an array of edge magnetic probes, we track the trajectory of single spheromaks ejected from one gun or the other. We determine a mean calibration constant for a given probe (all three pairs of collectors). We find that our smaller probe $\left(r=0.3 \mathrm{~cm}<\rho_{i}\right)$ has a calibration constant $\kappa=1.9$ \pm 0.4 . Our larger probe $\left(\mathrm{r}=0.6 \mathrm{~cm}>\rho_{\mathrm{i}}\right)$ has a calibration constant $\kappa=2.3 \pm 0.4$.

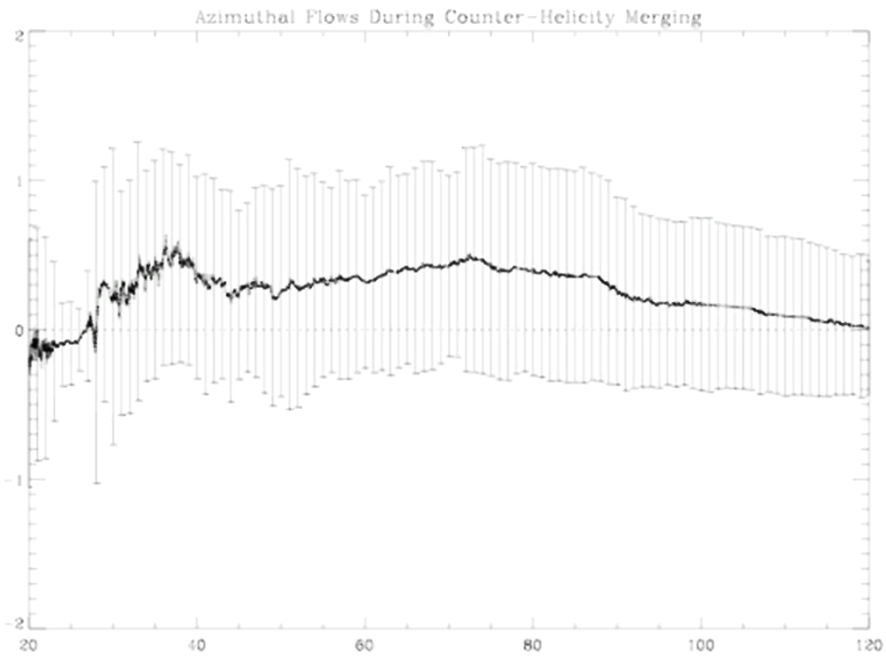

Figure 3. Local Mach probe measurement of outflow. Units are normalized to the sound speed (Mach number) Outflow speed, location, timing $(30-40 \mu \mathrm{s})$ are consistent with IDS data of figure 2

In Figure 3, we show the average flow in the azimuthal direction as measured by a Mach probe at the location we expect to see the maximum outflow $(\mathrm{r} / \mathrm{R}=0.8)$. The reconnection outflow in SSX is not purely radial so we expect to see an azimuthal component. Note that the reconnection site isn't coincident with the probe location on every shot making it necessary to average multiple shots together. Flow studies of single spheromaks show that the initial axial flow (up to $100 \mathrm{~km} / \mathrm{s}$ ) returns along the outer wall forming a large scale poloidal vortex. Single spheromaks have very little azimuthal flow or rotation.[5] 


\section{SOHO SUMER spacecraft measurement in chromosphere}

In a remarkable series of measurements, using the SOHO SUMER ultraviolet spectrometer, Innes et al monitored the dynamics of a $\mathrm{Si}_{\mathrm{IV}}$ line (1393 angstroms) to determine localized flow and heating during explosive reconnection events in the chromosphere $[15,16]$. The SUMER spectrometer had a spatial resolution of about 1 arcsecond (about $700 \mathrm{~km}$ on the solar disk) and a spectral resolution of about 0.044 angstrom (about $10 \mathrm{~km} / \mathrm{s}$ ). The entrance slit corresponded to a very small fraction of the solar surface $\left(10^{-5}\right)$ and was typically scanned in steps of about 1 arcsecond from east to west across the disk at 5 second exposures and eight positions. Scans at high solar latitude were sensitive to flow across the solar surface (nearly along the line of sight) while scans at the center of the disk were sensitive to upflows and downflows. Innes et al observed simultaneous red- and blue- shifts of the same line corresponding to bi-directional outflow due to reconnection in the chromosphere. Flow speeds up to $\pm 100 \mathrm{~km} / \mathrm{s}$ were measured.

\section{RECONNECTION HEATING}

Since spheromak merging generates a large $\nabla \times \mathbf{B}$, we expect strong current sheets and direct Ohmic electron heating within the reconnection volume.[6] In addition, the ion flows discussed above would be expected to viscously damp generating a population of heated ions.

\section{A. Electrons}

Model spectra for SSX plasmas have been calculated using an atomic physics code called PrismSpect[17]. Elemental composition has been estimated from vacuum ultraviolet spectroscopy. We observe emission mostly from carbon, with trace emission from oxygen and nitrogen. Soft $\mathrm{x}$-ray (SXR) emission from SSX is measured with a four-channel array of matched PIN photodiodes with thin $(100 \mathrm{~nm})$ metal filters $(\mathrm{Al}, \mathrm{Sn}, \mathrm{Ti}, \mathrm{Zr}$ ) in front of each. Chords for SXR and VUV are shown in Figure 1. Different filters have different passbands in the range 20-200 eV. Using model spectra, we have been able to interpret the SXR flux data to give a dynamical measurement of the electron temperature.

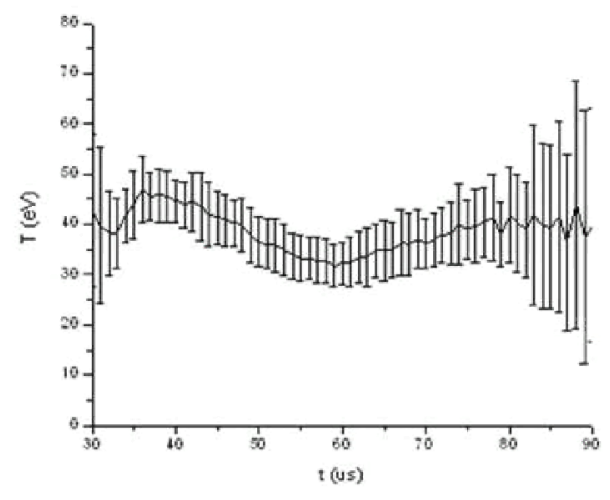

Figure 4. Electron heating measured with soft $x$-ray array. Heating occurs during reconnection activity $30-40 \mu$ s. 
In Figure 4, we show the electron temperature calculated from fits of SXR data to model spectra. Note that since the heated electrons are so mobile $\left(v_{e}=2.5 \mathrm{~m} / \mu \mathrm{s}\right)$ they rapidly leave the reconnection volume on newly reconnected field lines and disperse their heat throughout the entire SSX volume. Because of this, the signature of intense electron heating is dissipated in less than $1 \mu \mathrm{s}$. We have noticed that our highest energy x-ray bins have more signal than can be explained with a single temperature electron distribution function. In the future, we plan on modeling spectra using a second, higher temperature electron population or a harder electron tail. Baseline electron temperatures of $\mathrm{T}_{\mathrm{e}}=20 \mathrm{eV}$ have been independently confirmed by analyzing $\mathrm{C}_{\mathrm{III}} / \mathrm{C}_{\mathrm{IV}}$ line ratios $(97.7 \mathrm{~nm} / 155 \mathrm{~nm})$ measured with a VUV monochrometer with PrismSpect.

\section{B. Ions}

Ion temperature in SSX merging spheromaks has been measured with the ion Doppler spectrometer at the midplane.[9] Lineshapes from $\mathrm{C}_{\mathrm{III}}$ emission were measured during merging. Ion temperature extracted from the second moment of the lineshape data show heating up to $T_{i}=100 \mathrm{eV}$. Figure 5 shows the ion temperature as a function of time measured at the midplane of SSX. Note that the time of intense ion heating $(30-40 \mu \mathrm{s})$ is the same as the burst of Alfvenic outflow and electron heating noted above. This experiment was performed immediately after a session of He glow discharge conditioning of SSX. We find that our highest $T_{i}$ (and lowest densities) can be attained after the walls have been conditioned to remove adsorbed neutral gas.

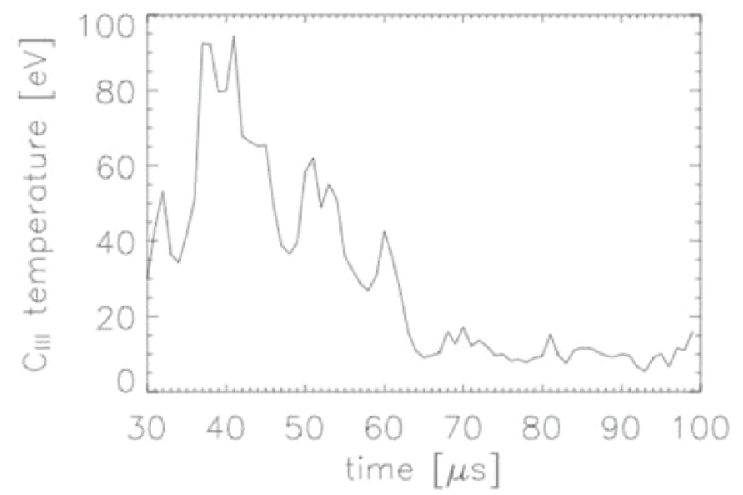

Figure 5. Ion heating measured with IDS. Heating occurs during reconnection activity $30-40 \mu$ s.

\section{Ion heating in the solar wind}

Ion Doppler techniques have also been employed to measure ion temperatures in the high corona and solar wind.[18] The UVCS/SOHO instrument has measured temperatures of high charge state ions (eg $\mathrm{O}_{\mathrm{VI}}$ ) within a few $\mathrm{R}_{\text {sun }}$ of the solar surface and has found ion temperatures that scale with the mass ratio $\left(T_{i o n} / T_{p}>M_{i o n} / M_{p}\right)$. For example, the temperature of $\mathrm{O}_{\mathrm{VI}}$ ions approaches $2 \times 10^{8} \mathrm{~K}$ at $3 \mathrm{R}_{\text {sun }}$ while the proton temperature is only $2 \times 10^{6} \mathrm{~K}$. In addition, proton temperatures are higher than electron temperatures $\left(T_{i}>T_{p}>T_{e}\right)$. This is similar scaling to what is seen in SSX. 


\section{QUADRUPOLE MAGNETIC FIELD}

In the two fluid model of magnetic reconnection, the ion and electron fluids decouple at the ion inertial scale but the magnetic field remains frozen to the electron fluid. The physical origin of the out-of-plane quadrupole can be understood either by considering the different trajectories of ions and electrons or by considering the dynamics of the electron fluid alone. In the latter argument, the electron fluid flows in a thin layer in the direction opposite the electric current. Since the magnetic field is frozen to the electrons, the electron fluid will tend to drag recently reconnected loops of magnetic field in the electron flow direction. The result is a quadrupolar structure of the magnetic field component in the direction of the electron flow.

In steady state, there will tend to be an inward directed $\mathrm{JxB}$ force supporting electron pressure $(\nabla \mathrm{P})$. If the Hall electric field $\mathbf{J x} \mathbf{B} / \mathrm{n}_{\mathrm{e}}$ is the dominant term in the generalized $\mathrm{Ohm}$ 's law, then $\mathrm{E}_{\text {Hall }}$ is the dominant part of the total electric field. Large inward directed electric fields have been observed both in space and in the lab.

\section{A. SSX laboratory measurement}

In order to better compare with space data and theoretical models, we rotate our 3D data to a coordinate system in which the magnetic field most closely resembles a standard 2D Xtype reconnection model. This is similar to the boundary normal or minimum variance coordinates of magnetospheric observations. Figure 6 illustrates magnetic data projected onto this plane. $[6,19,20]$ Note that in SSX this plane is canted relative to the axial direction of spheromak merging due to the twist in each flux tube associated with the toroidal and poloidal field components. Our inflow direction is along $\pm \mathbf{z}$. The data shown are from the spontaneous reconnection phase of a counter-helicity merging experiment at time $t=64 \mu \mathrm{s}$.

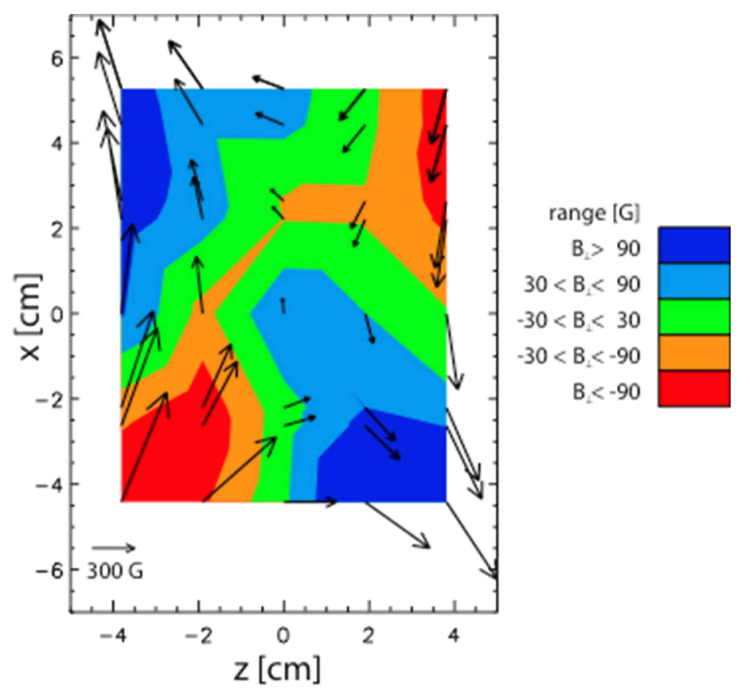

Figure 6. SSX quadrupole magnetic field. In-plane reconnecting magnetic field is represented as a vector field. 3D data are projected on a plane that best represents the idealized $2 \mathrm{D}$ geometry. SSX inflow direction is the $\pm \mathbf{z}$ direction. The scale is indicated with $300 \mathrm{G}$ magnitude vector below. Quadrupole field is represented as a color map. Magnitudes reach $\pm 150 \mathrm{G}$ near the outer reaches of the probe array. The ion inertial scale $\mathrm{c} / \omega_{\mathrm{pi}}$ is about $2 \mathrm{~cm}$ here. Two lobes of the quadrupole field are about 4 ion inertial lengths apart. 
There is a clear four-lobed quadrupolar structure to the out-of-plane magnetic field,as predicted on theoretical grounds for collisionless reconnection. To our knowledge, it is the first laboratory measurement of the out of plane magnetic quadrupole. Two lobes of the quadrupole field are about $8 \mathrm{~cm}$ or 4 ion inertial lengths apart. The interpretation is that the measured quadrupolar magnetic field having a magnitude of 100-150 Gauss (up to $25 \%$ of the in-plane field) is a consequence of the circulation of the Hall effect electric field $\mathbf{E}_{\mathrm{Hall}}=$ $\mathbf{J} \times \mathbf{B} / \mathrm{n}_{\mathrm{e}}$. This term comes into prominence when current sheets thin to about $\mathrm{c} / \omega_{\mathrm{pi}}$, and is a characteristic kinetic signature of reconnection in the low collisionality regime.

Figure 7 shows a map of the projection of the Hall electric field $\mathbf{J} \times \mathbf{B} / \mathrm{n}_{\mathrm{e}}$ onto the $2 \mathrm{D}$ reconnection plane defined by figure 6 from 3D probe measurements in SSX.[19,20] To our knowledge, it is the first laboratory measurement of the Hall electric field. We use an average electron density in the denominator of the expression. This map is highly similar to the inplane total electric field shown in Wygant et al (2005), inferred from Cluster data at a magnetotail tail crossing[21]. Note the important distinction that we measure the Hall electric field magnetically throughout the volume (from $\mathbf{J}, \mathbf{B}$, and $\mathrm{n}$ ) while in Cluster it is measured electrostatically along a chord. However, we have demonstrated that the Hall contribution dominates the electric field in the generalized Ohm's law in SSX.[11]

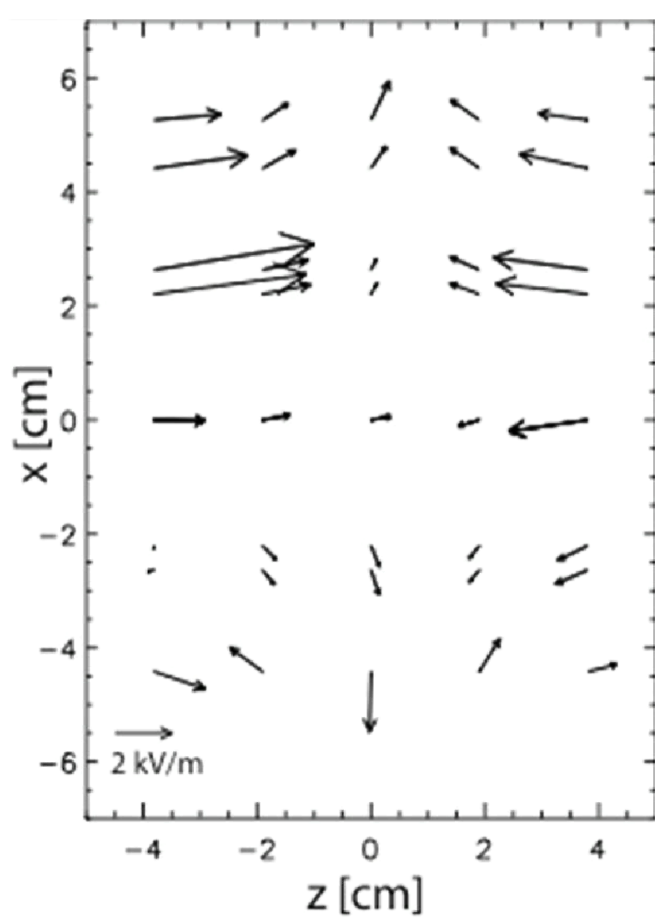

Figure 7. SSX Hall electric field. A vector map of the projection of the Hall electric field $\left(\mathrm{JXB} / \mathbf{n}_{\mathrm{e}}\right)$ onto the same plane as depicted in figure 6 . The Hall electric field is directed inward towards the current sheet and has a quadrupolar character. 


\section{B. Polar spacecraft measurement in the magnetopause}

Recent measurements by the Polar spacecraft at the earth's magnetopause have shown perhaps the clearest signature of kinetic magnetic reconnection in a single space observation to date. Data from the 28 second 1 April 2001 magnetopause crossing are presented Mozer et al. [22] The geometry of this crossing is fully 3D but the data are rotated and presented in the boundary normal coordinate or minimum variance system to accentuate the $2 \mathrm{D}$ reconnection geometry suggested by theory. It is assumed that the structure doesn't evolve during the 28 second transit of the reconnection layer. The reconnecting magnetic field clearly reverses sign from southward to northward, and the out-of-plane magnetic field clearly shows half of the characteristic quadrupole. At the magnetopause, the magnitude of the magnetic field nearly vanishes. By calculating the magnetopause speed, time can be converted to distance for the single spacecraft. Using the measured plasma density, distances can be expressed in units of ion inertial scale $\left(\mathrm{c} / \omega_{\mathrm{pi}}\right)$. The thickness of the magnetopause measured here is about 6 magnetosheath ion inertial scales. The two lobes of the quadrupole field are about 4 ion inertial lengths apart. It is interesting to note that the maximum ion outflow velocity was about $0.4 \mathrm{~V}_{\mathrm{A}}$ for this event (similar to the outflow observed in SSX, section II.A). Note also that Polar also observed an inward directed electric field correlated with the quadrupole (see figure 7)

\section{SUMMARY}

To summarize, several new experimental results are reported from spheromak merging studies at the Swarthmore Spheromak Experiment (SSX) with relevance to three-dimensional reconnection in laboratory and space plasmas. First, we report on recent velocity and temperature measurements of impurity ions using ion Doppler spectroscopy (IDS). Bidirectional outflow at nearly the Alfven speed is clearly observed. Similar observations have been reported in the solar chromosphere as well as the earth's magnetosphere. Second, electron heating is confirmed via SXR analysis and ion heating is measured with IDS. We observe all these signatures (plasma flow, ion and electron heating) coincident with a $3 \mathrm{D}$ magnetic reconnection event at $30-40 \mu$ s. Similar ion heating has been observed in the solar wind. Third, we discuss experimental measurement of the out-of-plane magnetic field near the reconnection zone showing a quadrupolar structure at the ion inertial scale. A similar quadrupolar structure has been observed at the earth's magnetopause also at the ion inertial scale. Also, we discuss a measurement of in-plane Hall electric field and non-ideal terms of the generalized Ohm's law in a reconnection volume of a weakly collisional laboratory plasma. Similar inward directed Hall electric field has been observed in the earth's magnetosphere (both at the magnetopause and magnetotail). Future plans include further studies of energetic electrons using SXR and a new, compact trapezoidal flux conserver for oblate FRC studies.

The authors gratefully acknowledge the technical assistance of S. Palmer and J. Haldeman at Swarthmore, and J. MacFarlane at Prism Computational Sciences.

\section{REFERENCES}

1. M.R. Brown, Physics of Plasmas 6, 1717 (1999)

2. P.M. Bellan, Spheromaks, Imperial College Press, (2000)

3. C.G.R. Geddes, T. W. Kornack, and M. R. Brown, Physics of Plasmas 5, 1027 (1998)

4. C.D. Cothran, A. Falk, A. Fefferman, M. Landreman, M.R. Brown, and M.J. Schaffer, Physics of Plasmas 10, 1748 (2003)

5. M.R. Brown, et al., Physics of Plasmas 13, 102503 (2006) 
6. M.R. Brown, C.D. Cothran, and J. Fung, Physics of Plasmas 13, 056503 (2006)

7. M. Landreman, C.D. Cothran, M.R. Brown, M. Kostora, and J.T. Slough, Rev. Sci. Instrum. 74, 2361 (2003)

8. M.R. Brown, C.D. Cothran, M. Landreman, D. Schlossberg, and W.H. Matthaeus, Astrophysical Journal Lett. 577, L63 (2002)

9. C.D. Cothran, J. Fung, M.R. Brown, and M.J. Schaffer, Rev. Sci. Instrum. 77, 063504 (2006)

10. C.D. Cothran, M. Landreman, W.H. Matthaeus, and M.R. Brown, Geophys. Res. Lett. 30, $1213(2003)$

11. C.D. Cothran, M. Landreman, M.R. Brown, and W.H. Matthaeus, Geophys. Res. Lett. 32, L03105 (2005)

12. T.W. Kornack, P.K. Sollins, and M.R. Brown, Phys. Rev. E 58, R36 (1998)

13. C.D. Cothran, J. Fung, M.R. Brown, and M.J. Schaffer, "Simultaneous Bi-directional Plasma Jets from a Laboratory Magnetic Reconnection Volume", Phys. Rev. Lett. submitted (2007)

14. I.H. Hutchinson, Physics of Plasmas 9, 1832 (2002)

15. D.E. Innes, B. Inhester, W.I. Axford, and K. Wilmelm, Nature 386, 811 (1997)

16. D.E. Innes, P. Brekke, D. Germerott, and K. Wilhelm, Solar Physics 175, 341 (1997)

17. J.J. MacFarlane, et al., Inertial Fusion Sciences and Applications, American Nuclear Society, p. 457 (2003)

18. S.R. Cranmer, Space Science Reviews, 101, 229 (2002)

19. W.H. Matthaeus, C.D. Cothran, M. Landreman, and M.R. Brown, Geophys. Res. Lett. 32, L23104 (2005)

20. M. Landreman, honors thesis, Swarthmore College (2003).

21. J.R. Wygant, C.A. Cattell, R. Lysak, et al., J. Geophys. Res. 110, A09206 (2005)

22. F.S. Mozer, S.D. Bale, and T.D. Phan, Phys. Rev. Lett. 89, 15002 (2002) 